\title{
SIMULATION CT-SCAN: A MARKETING PERSPECTIVE
}

\author{
Leonardo Chwif \\ Marcos Ribeiro Pereira Barretto \\ CEPI \\ Unifieo \\ R. Narciso Sturlini, 883 \\ Osasco , São Paulo, 06018-903, BRAZIL
}

\begin{abstract}
There is a general understanding in the simulation community that simulation is not reaching its full potential and is not widely used despite its well-known (among simulationists) benefits. This article's primarily intent is to discuss this belief, applying a marketing model whereby simulation is viewed as a product.
\end{abstract}

\section{INTRODUCTION}

In 1994, Khaled Mabrouk foresaw: "By the year 2000, simulation will replace spreadsheets as the analytical tool of choice for system analysis" (Mabrouk 1994). Now, 10 years later, we can affirm that this is far from being true. On Winter Simulation Conference 2003, during the panel session "Increasing Return on Investment from Simulation" (Gibson et. al 2003) the panelists raised several points for simulation not reaching its full potential as a decision making tool, being used just by a small part of potential users.

The purpose of this article is to go deeper into this discussion, shedding light on why simulation has not received a wider acceptance, pinpointing more elements in order to serve as a "CT-Scan" or diagnosis for taking actions to change this scenario.

This paper is organized as follows: Section 2 presents the results of a small market survey on some companies about their use of simulation and about courses in simulation. Section 3 presents a basic framework for marketing analysis which is McCarthy's $4 \mathrm{P} / 5 \mathrm{P}$. Section 4 applies the proposed framework to simulation viewed as a "product" immerse in the market. Finally, section 5 summarizes the main points and provides directions for future actions.

\section{MARKET RESEARCH RESULTS}

A market survey was conducted in approximately 30 Brazilian companies that claimed to use simulation because they were cited on web sites of simulation software vendors in
Brazil. Most of the responders came from technical areas (87\%) but there were also non technical responders, specifically from Marketing and related areas. Regarding the Profile of the Companies, it ranged from Agriculture, base industries, consumer product industries to services. The detailed profile of the companies researched and the responders is depicted in Figure 1. The question asked was: Are you still using simulation? The results were the following:

- $6 \%$ said simulation is not being used but they have it inside ERP system

- $2 \%$ are currently using simulation

- In $70 \%$ of the companies, simulation culture was completely lost (the person who was responsible for simulation has changed department or left the company)

- $22 \%$ knew that some simulation work had been done before but was not able to locate the person or the department

Another survey was conducted over the Internet, trying to address the question: "How many courses in simulation are being offered in MBA programs?" MBA programs were focused because the students of this kind of program have a higher maturity and can very rapidly become a simulation customer. Taking some of the top MBA programs in the world (Wharton, Harvard Business School, Columbia Business School, Stanford University, Insead, Sloan - MIT, London Business School, Kellog and University of Chicago), in $40 \%$ of them a course on discrete event simulation was offered. When considering all inter- net listed MBA programs, only $0.1 \%$ or 1 in 1000 MBA programs maintain a discrete event simulation course. This last research was based on a Goggle search for keywords therefore can only be taken as indicative <http: //www.google. com>.

The conclusion of these two researches is that simulation culture is being lost in Companies and is not widely discussed in MBA programs. 

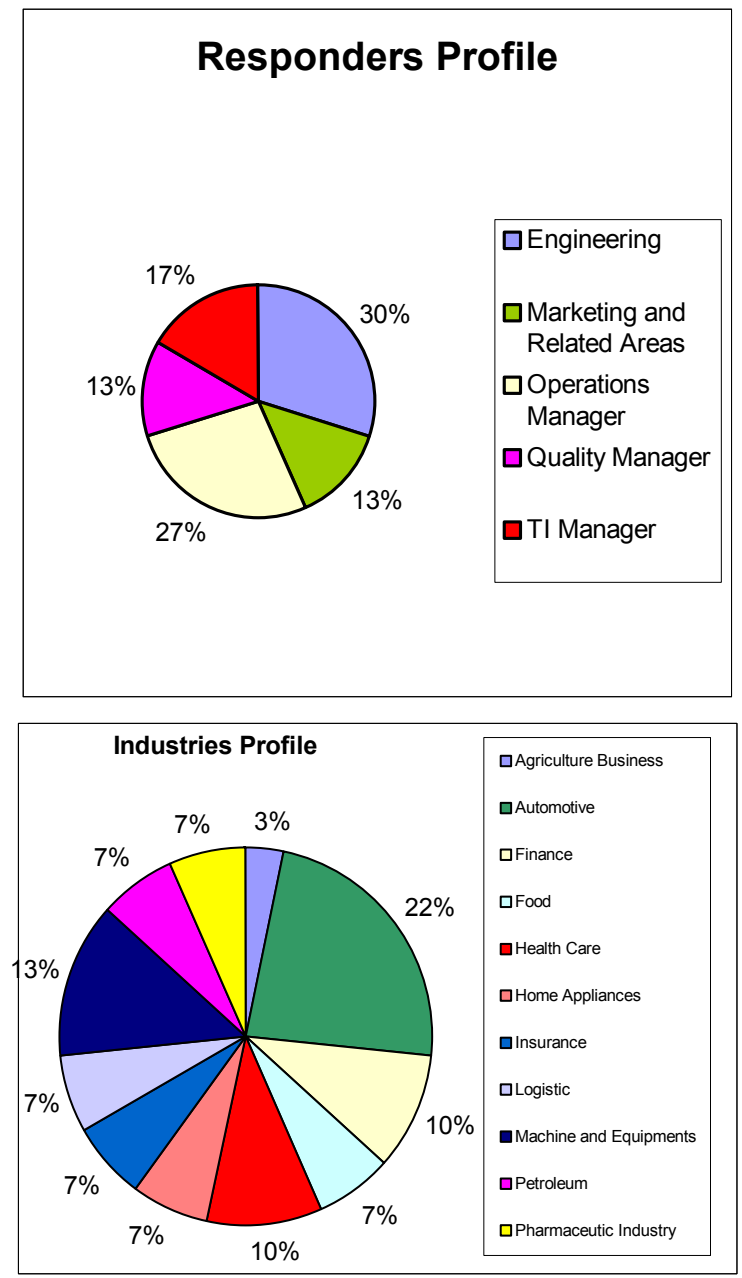

Figure 1: Profile of the Companies

\section{MCCARTHY'S 4P/5P MODEL}

To try to analyze the scenario exposed in the previous section, we will use McCarthy's 4P model for marketing strategic planning, which consists of analyzing:

- Product : are we delivering products that customers want?

- Price: are prices reasonable (in the customer's view) for what is being delivered?

- Place : are the products available at the right place, at the right time and in the right quantities?

- Promotion : which are the target groups? Are they informed/educated about the product?

When applied to services as is the case for the simulation market, McCarthy's model is augmented in one dimension, resulting in the $5 \mathrm{P}$ model.

- People : are our people well trained to deliver the product?
The next section will address simulation in each of these dimensions. It is important to clarify that simulation is taken here as the product: the simulation service provided to a company (ie, the service provided by an external company or by a internal person or team within the company).

\section{CT-SCANNING WITH MCCARTHY'S 4P/5P MODEL}

\subsection{Product}

This dimension will be addressed in three aspects: (1) customer expectation; (2) simulation concurrent products or substitutes and (3) product use.

Common sense suggests that a good product will sell itself. But History is full of counter-examples; take for instance, on automotive industry, Tucker and Edsel stories. Both cars were technically considered excellent but did not succeed in the marketing. As Young(1989) states: "The Edsel serves as a textbook example of corporate presumption and disregard for market realities. It also demonstrates that advertising and pre-delivery hype have their limits in inducing consumers to buy a new and unproven car". The central question is: is simulation the product customers are expecting? What do customers really want from simulation study?

Normally, she/he wants to make a good decision. Although simulation can offer other gains as the better understanding of the system, this is not customer's final objective when contracting a simulation study. Some customers contract a simulation study just for the fancy animation. For the sake of simplification our analysis will focus only on the "decision makers" type customers. Hence customers that views simulation as solely as an animation tool will not be considered in this paper.

As decision makers, customers have alternatives or concurrent products (to simulation) to aid them in the decision process. A customer may use a variety of methods formal or informal, including "guessing" (informal), simple or complex calculations (formal), queuing theory (formal), and so on. If there are alternatives or concurrent products, a rational customer will compare prices and time. The concurrent products for simulation as a formal/semiformal decision making tool, according Grabau (2001), are spreadsheets and process maps. By our experience a substantial amount of customers prefer informal methods (like guessing) of deciding that the formal ones (like simulation) methods: suppose for instance that a manager has to decide how many resources are needed for a desired level of production. He finds that 10 resources will be need but he asks for 12 , forecasting future expense cutting which will probably reduce workforce by $10-20 \%$. So what is the point of using a more formal dimensioning method? Even if he uses a working load spreadsheet the answer would be wrong at least from his point of view. 
But what would make a customer choose in favor of simulation? The simulationist's answer would be "because with simulation you can consider the randomness behavior of systems and the results will be more realistic". But how many customers really understand what randomness is? Lowery et al. (1994) reinforces this point : "Two reasons help to account for the slow acceptance of the use of simulation .... the other (reason) is managers continued dependence on deterministic decision making". In fact we found that managers who have knowledge of Six Sigma are eager to accept simulation as an important decision-making tool since the notion of randomness and variation is better understood.

Regarding the product use, simulation is not an indispensable tool in the sense that a decision maker must obligatory use it (in marketing terminology this can be viewed as a de-intensifier force). The other de-intensifier is that simulation is not being used in a continuous fashion. The majority of simulation work focuses either new systems or the optimization of existing systems (is mainly a technique for system planning and design). Simulation is not on daily, operational and continued work; we can say that the use of discrete event simulation is "discrete in time". For other uses of simulation in a more continuous way, the reader should refer to the article "Expanding Simulation Beyond Planning and Design" (Thompson 1994). Hence simulation is mostly used or driven when a change would be introduced in a system with the aim to see its impact. If the system operation remains the same (and there is no effort to make the system working better) simulation is not required.

\subsection{Price}

It is somehow a consensus that the cost of simulation is less than $2 \%$ of the cost of a project (Banks 2000). This figure is neither negligible in absolute terms (it can vary from USD $10 \mathrm{~K}$ to $100 \mathrm{~K}$ ), nor as relative to project cost, since that net profit from many industries lies within these numbers. Therefore, the argument that "simulation's relative price is low" does not hold up. So it may be better not to use isolated cost and apply other techniques such as ROI (Return on Investment), having a simulation study as a part of the investment itself. Several cases studies in literature proved the value of simulation (Banks 2000) and the gains that it provided (in many cases several more times that the amount invested in simulation). But it is very hard (if not impossible) to estimate ROI of a simulation study before actually finishing the study. Gray (1976) affirms: "If the benefits of simulation are known precisely beforehand, it usually implies that the outcomes of the simulation are known beforehand, in which case the simulation is not needed". Banks (2000) tried to deal with this problem by thinking of simulation as "insurance" but even in this case it is not straightforward to "put a value on simulation".

\subsection{Place}

Unfortunately, simulation results are not there when they are needed. Currently, simulation is considered a very time consuming process due to the inherent steps needed to construct the mental model, construct the conceptual model, get the data, analyze the data, construct the computerized model, verify and validate the model, get the results of experiments and so on. According to Keller, Harrel and Leavy (1991) "..Simulation can be intensely time consuming and the best approach is to assume that almost any model will take you twice as long to complete than you expected". McLean and Leong (2001) states: "The development and maintenance of models of their production system and resources is very costly. For example, the development of a detail simulation model of a single machine tool may take an engineer 4 to 6 weeks". The long timeframe for a simulation study acts also as a de-intensifier of the Simulation Product.

\subsection{Promotion}

Who actually is selling simulation?

a) Academia

b) Software vendors

c) Independent Consultants

d) Internal Selling (by people who works with simulation within a company)

As we stated in section 2 academia promotes simulation in very small number of MBA courses, normally sharing a discipline with other subjects. This issue could be seen as a "chicken and egg" problem: if the market is not widely applying simulation it is pointless to offer more simulation courses. If simulation courses are not offered, the people will not know better this technique and will use it less. This negative cycle must be broken.

Most of software vendors are primarily focusing on the promotion of a simulation tool rather than on the promotion of simulation itself. As any tool vendor, they generally sell the idea that "the tool will solve your problem". It is the same thing as saying: "Anyone with a very good pair of scissors can do a very good hair-cut", which is not true. Most independent consultants play a similar role because they are representatives or generally use a very specific simulation tool. Even the best simulation software, when in an unskilled and uneducated (in a simulation sense) modeler's hand, will deliver a low quality product. But a skilled professional can do acceptable work with a simple tool. Paraphrasing Harrel and Tumay (1995): “A fool with a tool is still a fool". According to Gibson et al. (2003) "...the Simulation Community may have accidentally hurt itself by overstressing the importance of "ease of use" software, and not stressing the importance of "engi- 
neering discipline" - training and experience - need to properly employ such software, manage the project, and produce correct and appropriate results". Therefore simulation software vendors play a key role in the success or failures of the simulation market.

Finally, we have the internal seller. The market research in section 2 showed this reality: for most surveyed companies, simulation internal culture was lost because the person responsible for simulation was in another department or had moved to another job. This is also the personal experience of one of this paper's authors, who used to work for a large home appliance company. Besides this, a specialist in simulation also normally shows little selling skill, due to her/his strong technical background, which makes internal promotion even weaker.

\subsection{People}

The modeler (also called "simulation analysts") in simulation plays a key role in delivering a good product, or in other words, satisfying the clients. Jerry Banks and Matt Rohrer wrote an article addressing the required skills of a Simulation Analyst to conduct a successful simulation study (Rohrer and Banks 1998). According to them skills were broken down into required skills, desired skills and acquired skills for each component. For instance the required skills include an ability to see details in a system, possessing systems understanding, and having the capability to perform technical writing. The analyst should be analytical, a logical thinker, well organized and blessed with a good memory. Besides these skills, a modeler should have some specific knowledge basis or background. Keller, Harrel and Leavy (1991) defined the "four education pillars of successful simulation" as being Statistics, Experimental Design, Process and Product knowledge, and Logic. The former and the second "pillar" is used to gather and analyze the input data correctly, plan the simulation experiments and analyze properly the outcomes from simulation. The Modeler should understand how things works otherwise he will not be able to correctly model the process. He also needs to determine the appropriate level of detail of the model and its scope. The fourth pillar is "logic" and it is more required at the implementation phase of a simulation study, i.e. the construction of the computerized model. This is also strongly correlated to abstract thinking.

As can be seen, we could make an entire article discussing the skills, competences and abilities of the modeler, but the question that arises from this discussion is: "Do we have good modelers in the market?". We fear that the answer may be 'no'. In fact, according to John Carson, "Good simulation analysts are hard to find". (Banks 1998). And more: "More than half of the (simulation) studies that I have seen fall short of acceptance and implementation failed as a direct result of poor procedure" (Lowery et. al. 1994).
This causes another question to be raised: "What happens when an unsuccessful simulation project is conducted" by a "bad" modeler? Banks were assertive in answering this question: "If the project is successful, then the money is well spent and will promote the technology. If the project is not successful, it hurts the reputation of simulation and, by association, each of us....Do not commit to a simulation project if...there is a real chance that it can not be properly completed." (Banks and Gibson 1997). Again, this acts as a de-intensifier in the Product dimension, resulting in a negative recall of simulation service level.

\section{SUMMARY AND DIRECTIONS FOR FUTURE WORK}

The main purpose of this article is to make a CT-Scan or diagnosis for the less-than-expected growth of simulation in the market. In order to do this, we took a marketing approach by identifying the 5 Ps of Simulation Product according to McCarthy's Marketing Framework. It started with a marketing research on companies who claimed to work with simulation and about simulation application in MBA's courses. The mains points of this reseach were summarized below:

1. Only $2 \%$ of the companies that claimed to apply simulation is really using it

2. A small fraction of MBA courses world wide is really teaching discrete event simulation as part of its program.

By these two points the main conclusion is that simulation culture is being lost in companies that used to apply simulation and is not widely taught in MBA programs. The possibly causes of these effects were pinpointed also in this article. Below a summary of them:

1. It is clear that the majority of us from the simulation community are great defenders of simulation. However we should address or focus on the customer. Even the best product can fail to meet the customers' needs (remember the case of Tucker and Edsel cars). We must not become presumptive and disregard market realities or customers' needs.

2. The modeler plays a key role in the success of simulation. If one simulation study succeeds, there is a ground for further simulations studies. Where are good modelers being formed?

3. Are we selling simulation as a whole or are we just selling simulation software? In the selling process are we addressing exactly the customers' needs or what we think is important for simulation? Are we substituting the entire simulation process by simulation software? 
It was not the intention of this article to propose actions to alter this scenario; this is left to the simulation community. But it is clear from a marketing perspective that a set of actions must be taken if we want to see a substantial growth of simulation.

\section{ACKNOWLEDGMENTS}

The authors would like to thank the Brazilian's funding agencies (CAPES, CNPQ and FAPESP) for their support to national research. The authors would also like to thank Cristina Kano for the help on the Marketing Survey.

\section{REFERENCES}

Banks, J. 1998. Simulation Specialists Thrive in Consulting, Industrial Engineer, 30(10):20-21.

Banks, J. 2000. Putting a Value on Simulation, Industrial Engineer, 32(8): 18 .

Banks, J. and R, Gibson. 1997. Don't Simulate When..., Industrial Engineer, 29(9):30-32.

Gibson, R., D. J. Medeiros, A. Sudar, B. Waite and M. W. Rohrer. 2003. Increasing Return on Investment from Simulation (Panel). In Proceedings of the 2003 Winter Simulation Conference, ed. S. Chick, P.J. Sanchez, D. Ferrin, and D.J Morrice, 2027- 2032. Piscataway, New Jersey: Institute of Electrical and Electronics Engineers.

Grabau, M.R. 2001. Averages Kill (or how to sell business process simulation). In: Proceedings of the $2001 \mathrm{Win}$ ter Simulation Conference, ed. B.A. Peters, J.S. Smith, D.J. Medeiros, and M.W. Rohrer, 1262-1265. Piscataway, New Jersey: Institute of Electrical and Electronics Engineers.

Gray, P. 1976. The Economics of Simulation. In: Proceedings of the 1976 Winter Simulation Conference, ed. H.J. Highland, T.J.Schriber, and R.G. Sargent, 17-25. Piscataway, New Jersey: Institute of Electrical and Electronics Engineers.

Harrel, C. and K. Tumay. 1995. Simulation Made Easy: A Managers Guide, Engineering \& Management Press.

Keller L., C. Harrel, and J. Leavy . 1991. The Three Reasons why Simulation Fails, Industrial Engineer, 23(4):27-31.

Lowery, J.C, B. Hakes, W. L. Lilegdon, L. Keller, K. Mabrouk and F. McGuire. 1994. Barriers to implementing Simulation in Health Care, In:Proceedings of the 1994 Winter Simulation Conference, ed. J.D. Tew, S. Manivannan, D.A. Saqdowski, and A.F. Seila, 868-875. Piscataway, New Jersey: Institute of Electrical and Electronics Engineers.

Mabrouk, K. 1994. Mentorship: A Stepping Stone to Simulation Success, Industrial Engineer, 26(2):41-43.

McLean, C and S. Leong. 2001. The Expanding Role of Simulation in Future Manufacturing System. In: Proceedings of the 2001 Winter Simulation Conference, ed. B.A Peters, J.S Smith, D.J Medeiros, and M.W. Rohrer, 1478-1486. Piscataway, New Jersey: Institute of Electrical and Electronics Engineers.

Rohrer, M and J. Banks. 1998. Required Skills of a Simulation Analyst, Industrial Engineer, 30(5):20-23.

Thompson, M.B. 1994. Expanding Simulation Beyond Planning and Design, Industrial Engineer, 26(10):64-66.

Young, A. 1989. The Rise and Fall of Edsel, The Freeman (A publication for Economic Education Inc), 38(9). Available online via <http://www. fee. org/vnews . php?nid=2115> [accessed January 31, 2004].

\section{AUTHOR BIOGRAPHIES}

LEONARDO CHWIF graduated in Mechanical Engineering (Mechatronic Specialization) in 1992 at the University of Sao Paulo and got his M.Sc. degree in 1994 and his Ph.D. in Simulation in 1999 from the same University. He spent a session at Brunel university as a research visitor at the Centre for Applied Simulation Modelling. Upon graduation Dr. Chwif joined the Brazilian branch of Mercedes-Benz truck manufacturer and then he joined the Brazilian branch of Whirlpool Corporation. Currently he is C.E.O of Simulate Simulation Technology. Dr. Chwif also teaches an introductory graduate simulation course at Mauá School of Engineering and a simulation course at specialization level at University of São Paulo. He also a teacher and researcher with CEPI (Information Technology Research Center) at Centro Universitário FIEO (Unifieo). His email is <leochwif@ usp.br>.

MARCOS RIBEIRO PEREIRA BARRETTO graduated in Electronic Engineering in 1983. He got his M.Sc. degree in 1988 and his Ph.D. in 1993, both from the University of Sao Paulo. He is a professor and researcher with CEPI (Information Technology Research Center) at Centro Universitário FIEO (Unifieo). His email is $<$ mrpbarre@usp.br>. 